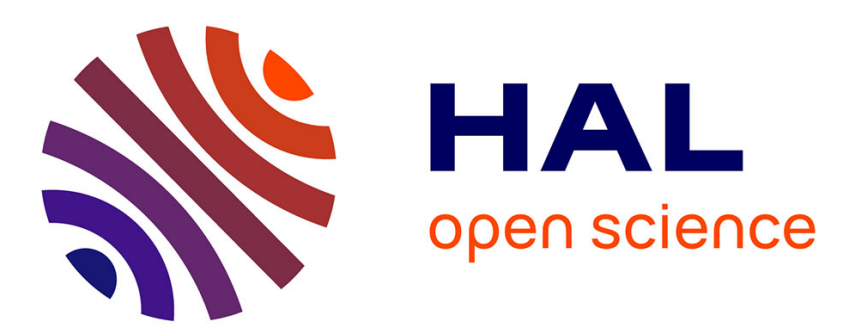

\title{
New degrees of freedom for high-order Whitney approximations of Darcy's flows
}

\author{
Ana Alonso Rodríguez, Francesca Rapetti, Elena Zappon
}

\section{To cite this version:}

Ana Alonso Rodríguez, Francesca Rapetti, Elena Zappon. New degrees of freedom for high-order Whitney approximations of Darcy's flows. Numerical Algorithms, In press, 10.1007/s11075-02001022-4 . hal-02948033

\section{HAL Id: hal-02948033 https://hal.science/hal-02948033}

Submitted on 6 Oct 2020

HAL is a multi-disciplinary open access archive for the deposit and dissemination of scientific research documents, whether they are published or not. The documents may come from teaching and research institutions in France or abroad, or from public or private research centers.
L'archive ouverte pluridisciplinaire HAL, est destinée au dépôt et à la diffusion de documents scientifiques de niveau recherche, publiés ou non, émanant des établissements d'enseignement et de recherche français ou étrangers, des laboratoires publics ou privés. 


\title{
New degrees of freedom for high-order Whitney approximations of Darcy's flows
}

\author{
Ana Alonso Rodríguez • \\ Francesca Rapetti · Elena Zappon
}

Received: date / Accepted: date

\begin{abstract}
We consider the flow of a viscous incompressible fluid through a rigid homogeneous porous medium. We propose a high-order discretisation based on Whitney finite elements, namely, Raviart-Thomas finite elements of degree $r+1$ for the discharge and discontinuous piecewise polynomial finite elements of degree $r$ for the pressure, with $r \geq 0$. We comment on the use of new degrees of freedom that have a clear physical meaning, the so-called weights on the small simplices, for the involved discharge and pressure fields. We describe a new numerical strategy to solve the discrete problem based on a tree-cotree block-decomposition of the unknowns, that is natural when considering these new degrees of freedom. Preliminary numerical tests in two dimensions confirm the stability of the adopted method and the effectiveness of the new degrees of freedom.
\end{abstract}

Keywords Darcy equation · Whitney finite elements · high order · new degrees of freedom $\cdot$ graph theory $\cdot$ exterior differential calculus

Mathematics Subject Classification (2010) 65N30 - 76M10 - 05C05

\author{
Ana Alonso Rodríguez \\ Dipartimento di Matematica, Università degli Studi di Trento \\ Via Sommarive, 14, I-38050 Povo \\ Phone: +390461 281512 \\ E-mail: alonso@science.unitn.it \\ Francesca Rapetti \\ Dép. de Mathématiques J.-A. Dieudonné, Université Côte d'Azur \\ Parc Valrose, F-06108 Nice \\ Phone: +33 (0)4 89150540 \\ E-mail: Francesca.RAPETTI@univ-cotedazur.fr \\ Elena Zappon \\ Dipartimento di Matematica MOX Politecnico di Milano \\ Via Bonardi 9, I-20133 Milano \\ Phone: +3902 23994611 \\ E-mail: elena.zappon@polimi.it
}




\section{Introduction}

In this paper, we consider Darcy's problem in the framework of the finite element exterior calculus and propose new degrees of freedom for the high order mixed finite element discretisation of this problem on simplicial meshes.

Darcy's problem describes the flow of a viscous incompressible fluid in a porous medium. It is a well-known problem from the mathematical point of view (see, e.g., [11], [28]), and in scientific journals, it is among the most studied ones, often coupled with either the heat equation or fracture mechanics. Darcy's problem is chosen on purpose here, the idea underlying this work is indeed to show that the documented mathematical pitfalls characterising this problem can be straightforwardly avoided by relying on compatible approaches as those suggested by the finite element exterior calculus (FEEC).

The FEEC uses tools from differential geometry and homological algebra to define, or suggest, the finite element discretisation that is the most compatible with the geometric and topological structure of the PDE problem under exam (see, e.g., [8], [9], [10] and the references cited therein). By reformulating the continuous problem in terms of differential forms, one gets some indications on the discrete formulation with a choice of finite element spaces that is natural, only dictated by the nature of the involved fields and by the mathematical structure of the considered problem. More specifically, the choice of the degrees of freedom that are suggested by the FEEC approach reflects the nature (and global regularity properties) of the fields they represent. When structural features of the continuous problem are preserved at the discrete level, as it is the case with compatible discretisations, stability and consistency properties required for the convergence are guaranteed. This is detailed in the present work for the considered problem and supported by numerical results.

Natural finite element spaces suggested by the FEEC approach are Whitney's differential $k$-forms $\mathcal{P}_{r+1}^{-} \Lambda^{k}(\mathcal{T})$ of polynomial degree $r+1$ [10], that are the Raviart-Thomas $R T_{r+1}($ for $k=2)$ and the discontinuous $D P_{r}$ (for $k=3$ ) FE spaces, respectively, when passing to vector formalism [28]. Here we rely on these FE spaces with a new set of degrees of freedom on simplices, firstly introduced in [29]. For a $k$-form $w \in \mathcal{P}_{r+1}^{-} \Lambda^{k}(\mathcal{T})$, classical degrees of freedom are the traditional moments (see, e.g., [9]). The new degrees of freedom are integrals of $w$ on small $k$-simplices, a set of subsimplices obtained by suitable contractions of the original mesh elements. These contractions are homothetic transformations on the space, namely the new subsimplices have smaller size while keeping the same shape and orientation of the original mesh elements. This allows for adopting degrees of freedom that reflect the nature of the fields they are associated with and to preserve the meaning of degrees of freedom as cochains, for $r>0$, in the spirit of Whitney's original work [35] dating back to 1957. For $r=0$, this is known since the 80s, when Bossavit made the connection between Whitney's forms $\mathcal{P}_{1}^{-} \Lambda^{k}(\mathcal{T})$ used by geometers and the first kind of Nédélec $H$ (curl) and $H$ (div) conforming elements used (see, e.g.,[14]). By choosing the weights as degrees of freedom for finite element discretisations, we can adopt, in fluid mechanics, techniques that are well-known in electro- 
magnetism. Here, it is the case of a tree-cotree block-wise decomposition, as the one proposed in [7] for $r=0$, to impose the divergence free constraint and generalise it to the case $r>0$ of high order approximations. This new tree-cotree approach results in a smaller system to be solved for the discharge unknown.

The paper is organised as follows:

- In Section 2, we formulate Darcy's problem in terms of differential forms. This is a delicate step where the involved physical phenomenon matters to determine the nature of the fields at play. The FEEC approach then guides the discrete formulation. If $n$ denotes the dimension of the physical domain $\Omega$ where the Darcy's problem is set and $\mathcal{T}$ is a simplicial mesh over $\bar{\Omega}$, the pair of spaces $\mathcal{P}_{r+1}^{-} \Lambda^{n-1}(\mathcal{T})$, of the Raviart-Thomas $H$ (div)-conforming finite elements of degree $r+1$, for the approximation of the discharge, and $\mathcal{P}_{r}^{-} \Lambda^{n}(\mathcal{T})$, of the discontinuous piecewise polynomial elements of degree $r$ for the $L^{2}$-approximation of the fluid's pressure pop up naturally.

- In Section 3, we write, in vector formalism, the discrete formulation suggested by the FEEC approach of the previous section. We recall the stability and consistency of the FE approach and give its matrix formulation.

- In Section 4, we introduce the new degrees of freedom, the weights, defined in [29], and the basis functions for the high order mixed finite element spaces involved in the discretisation of Darcy's equations.

- The next three sections are dedicated to numerical results. In Section 5, we focus on the numerical approximation of the inf-sup constant. We check, both in $h$ and in $r$, its convergence to the continuous value for a model problem. The choice of degrees of freedom (and thus of the basis functions in duality with them) does not influence its value but the way how the divergence free constraint is imposed. This latter aspect is discussed in Section 6 where a tree-cotree decomposition is used to address this issue. Section 7 includes some considerations about the computational complexity of the proposed approach, that could be introduced in existing software packages to reduce the cpu time for the solution of the discrete problem.

- Finally, in Section 8, we recap the methodology adopted in the paper and draw some conclusions on the basis of the results of the numerical tests.

\section{Darcy's problem governing equations}

We recall the governing equations of Darcy's stationary flows and comment on the nature of the involved fields (velocity, pressure, ...) in the FEEC framework.

Let $\Omega$ be a bounded Lipschitz polyhedral domain of $\mathbb{R}^{n}$, with $n \geq 1$. The experimental law formulated in 1856 by the French hydraulic engineer Henry P. G. Darcy (1803-1858) in [18] can be written in terms of the instantaneous discharge rate $\mathbf{q}\left([L][T]^{-1}\right)$ of the fluid through $\Omega$ as

$$
\mathbf{q}=-\frac{\kappa}{\nu} \operatorname{grad}(h g)
$$


where $\kappa\left([L]^{2}\right)$ is the intrinsic permeability of the medium in the domain, $\nu$ $\left([L]^{2}[T]^{-1}\right)$ the kinematic viscosity of the fluid flowing through the domain, $g$ $\left([L][T]^{-2}\right)$ is the acceleration due to gravity and $h([L])$ the hydraulic head.

In this form it is very similar to Ohm's law for conductors $J=-\sigma \operatorname{grad} V$ where the electric conductivity $\sigma$ is in fact a Hodge operator from the space of differential 1-forms $\Lambda^{1}(\Omega)$ to the space of differential 2-forms $\Lambda^{2}(\Omega)$. The hydraulic potential $(h g)$ (resp., the discharge rate q) acts like the voltage $V$ (resp., the current $\mathbf{J}$ ) and the constant $\kappa / \nu$ is the hydraulic conductivity that describes the fluid/medium as the electric conductivity $\sigma$ characterises the conductor. We now replace the hydraulic potential by the expression $h g=\frac{p}{\rho}+$ $z g$ where $p\left([M][L]^{-1}[T]^{-2}\right)$ is the pressure at the chosen point, $\rho\left([M][L]^{-3}\right)$ is the density of the fluid and $z([L])$ is the elevation of the point above a reference plane, with the positive $z$-direction pointing upward (so in the direction opposite to the gravitational acceleration). We thus get

$$
\mathbf{q}=-\frac{\kappa}{\nu} \operatorname{grad}\left(\frac{p}{\rho}\right)+\frac{\kappa}{\nu} \mathbf{f}
$$

where $\mathbf{f}=-\operatorname{grad}(z g)$ is associated with the additional external potential $(z g)$ due to the elevation $z$. Equivalently one has

$$
\frac{\nu}{\kappa} \mathbf{q}=-\operatorname{grad}\left(\frac{p}{\rho}\right)+\mathbf{f} .
$$

The problem's formulation in terms of differential forms, as explained in Fig. 1, makes use of two well known operators: one is the exterior derivative d, necessary to compute derivatives, like gradients, divergences, or curls; the other is the Hodge star $*$ that takes into account the properties of the specific media. The fluid discharge $\mathbf{q}$ is a 2 -form as it indicates the mass of fluid transported across a given surface area per unit time. The hydraulic potential is a 0 -form, a scalar function that can be evaluated at any point of the medium. The key point is that these two forms do not live on the same complex. Once introduced the operators $\mathrm{d}$ and $*$, we can organise the information in a Tonti's type diagram [34], as the one in Fig. 1. The primal complex (resp. dual complex) is drawn vertically on the left (resp. right) and we move up-down (resp. downup) with the operator d. The two complexes are linked by horizontal lines, one line for each Hodge operator. In Darcy's law, the two forms q and dV are related by the Hodge operator $* \frac{\nu}{\kappa}$. Moving vertically upward, from $\mathbf{q}$, by applying $\mathrm{d}$ we get $s$, namely $\mathrm{d} \mathbf{q}=s$. Darcy's problem thus reads

$$
*_{\nu / \kappa} \mathbf{q}+\mathrm{d}\left(*_{1 / \rho} p\right)=\mathbf{f}, \quad \mathrm{d} \mathbf{q}=s .
$$

Note that the $\mathrm{d}$ contained in the left-hand equation acts on a 0 -form whereas the $\mathrm{d}$ appearing in the right-hand equation acts on a $(n-1)$-form. In $\mathbb{R}^{3}$ with the standard metric they correspond to the grad operator on scalar fields and the div operator on vector fields respectively. From (1), we have

$$
\int_{\Omega}\left(*_{\nu / \kappa} \mathbf{q}+\mathrm{d}\left(*_{1 / \rho} p\right)\right) \wedge \mathbf{v}=\int_{\Omega} \mathbf{f} \wedge \mathbf{v},
$$




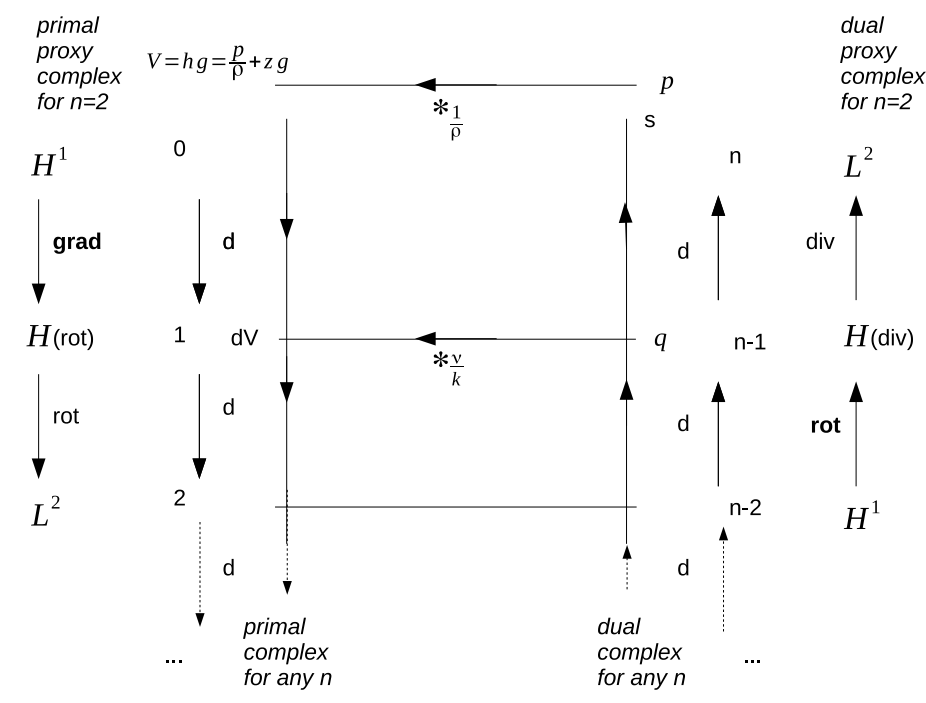

Fig. 1 Tonti's diagram for Darcy's law in terms of differential forms. On the vertical left and right sides of the diagram, we indicate, for $n=2$, the primal and dual proxy complexes, i.e., the two de Rham sequences of functional spaces (see, e.g., their definitions in [11]), associated with Darcy's law in vector formalism. When $n=2$, the curl operator is generally denoted either rot when applied to a vector field $\mathbf{v}=\left(\mathbf{v}_{1}, \mathbf{v}_{2}\right)^{T}$ (we have $\left.\operatorname{rot} \mathbf{v}=\partial_{x_{1}} \mathbf{v}_{2}-\partial_{x_{2}} \mathbf{v}_{1}\right)$ or rot when applied to a scalar field $v$ (we have $\left.\operatorname{rot} v=\left(\partial_{x_{2}} v,-\partial_{x_{1}} v\right)^{T}\right)$.

$$
\int_{\Omega} \mathrm{d} \mathbf{q} \wedge *_{1 / \rho} \psi=\int_{\Omega} s \wedge *_{1 / \rho} \psi
$$

Here $\mathbf{v}$ and $\psi$ are a $(n-1)$-form and a $n$-form respectively. Moreover, since $\left(*_{1 / \rho} p\right)$ is a 0 -form, the integration by parts' formula yields

$$
\int_{\Omega} \mathrm{d}\left(*_{1 / \rho} p\right) \wedge \mathbf{v}=-\int_{\Omega} *_{1 / \rho} p \wedge \mathrm{d} \mathbf{v}+\int_{\partial \Omega} \operatorname{Tr}\left(*_{1 / \rho} p\right) \wedge \operatorname{Tr} \mathbf{v} .
$$

Hence equations (2) and (3) (for $s=0$ ) read

$$
\begin{gathered}
\int_{\Omega} *_{\nu / \kappa} \mathbf{q} \wedge \mathbf{v}-\int_{\Omega} *_{1 / \rho} p \wedge \mathrm{d} \mathbf{v}=\int_{\Omega} \mathbf{f} \wedge \mathbf{v}-\int_{\partial \Omega} \operatorname{Tr}\left(*_{1 / \rho} p\right) \wedge \operatorname{Tr} \mathbf{v} \\
\int_{\Omega} \mathrm{d} \mathbf{q} \wedge *_{1 / \rho} \psi=0 .
\end{gathered}
$$

The FEEC approach guides the discrete formulation. We decompose $\Omega$ into a finite set $\mathcal{T}$ of $n$-simplices. For $r \geq 0$, let $\mathcal{P}_{r+1}^{-} \Lambda^{k}(\mathcal{T})$, with $k \in\{0,1, \ldots n\}$, be the spaces of Whitney's differential $k$-forms [35] of polynomial degree $r+1$. For $0 \leq k \leq n$, it is possible to connect these spaces of differential forms with finite element spaces of scalar and vector functions [12], thank to the pioneering work, done by Bossavit in the early 80s (see [14] details). When 
$n=3$, the set $\mathcal{P}_{r+1}^{-} \Lambda^{1}(\mathcal{T})$ and $\mathcal{P}_{r+1}^{-} \Lambda^{2}(\mathcal{T})$ corresponds, respectively, with the $H$ (curl) and $H$ (div) conforming finite element spaces of degree $r+1$, belonging to the first Nédélec's family [27]. For $n=2$, the set $\mathcal{P}_{r+1}^{-} \Lambda^{1}(\mathcal{T})$ correspond with the $H$ (div)-conforming finite element space of degree $r+1$, firstly introduced in [31]. The pair of spaces $\mathcal{P}_{r+1}^{-} \Lambda^{n-1}(\mathcal{T})$, of the Raviart-Thomas $H$ (div)-conforming finite elements of degree $r+1$, for the approximation of the discharge, and $\mathcal{P}_{r+1}^{-} \Lambda^{n}(\mathcal{T})$, of the discontinuous piecewise polynomial elements of degree $r$ for the $L^{2}$-approximation of the fluid's pressure pop up naturally among other possible pairs.

We have naturally obtained the mixed finite element discretisation of Darcy flow following the dual complex. Following the primal complex, Darcy flow can be rewritten in terms of the hydraulic potential and this potential discretised in $\mathcal{P}_{r+1}^{-} \Lambda^{0}(\mathcal{T})$ that corresponds to using Lagrange finite elements. Both approaches involve only the original (primal) mesh $\mathcal{T}$ of $\Omega$. An alternative discretisation adopting also the dual mesh has been proposed in [24] using the discrete exterior calculus (DEC).

\section{Whitney finite elements for the Darcy problem}

We recall in this section some well-known results concerning the mixed finite element approximation of the Darcy's problem solution (see, e.g., [28]). We consider the following boundary value problem:

$$
\begin{cases}\frac{\nu}{\kappa} \mathbf{q}+\operatorname{grad}\left[\frac{1}{\rho} p\right]=\mathbf{f} & \text { in } \Omega \\ \operatorname{div} \mathbf{q}=0 & \text { in } \Omega \\ \mathbf{q} \cdot \mathbf{n}=0 & \text { on } \Gamma_{N} \\ \frac{1}{\rho} p=H_{D} & \text { on } \Gamma_{D}\end{cases}
$$

where $\mathbf{n}$ is the unit outward normal to $\partial \Omega$ and $\Gamma_{N}, \Gamma_{D}$ are open disjoint subsets of $\partial \Omega$ such that $\partial \Omega=\overline{\Gamma_{N}} \cup \overline{\Gamma_{D}}$. The function $\mathbf{f} \in\left(L^{2}(\Omega)\right)^{n}$ is known, being $L^{2}(\Omega)$ the space of measurable, square integrable functions on $\Omega$, together with $H_{D} \in H^{1 / 2}\left(\Gamma_{D}\right)$. For the latter, we set $H^{1}(\Omega)=\left\{q \in L^{2}(\Omega), \operatorname{grad} q \in\right.$ $\left.\left(L^{2}(\Omega)\right)^{n}\right\}$ and $H^{1 / 2}(\partial \Omega)$ denotes the image in $L^{2}(\partial \Omega)$ of the trace operator $\gamma$ acting on functions of $H^{1}(\Omega)$. The space $H^{1 / 2}\left(\Gamma_{D}\right)$ contains the restrictions to $\Gamma_{D}$ of the functions belonging to $H^{1 / 2}(\partial \Omega)$. Let us also consider the following functional spaces:

$$
\begin{aligned}
& H(\operatorname{div} ; \Omega)=\left\{\mathbf{v} \in\left(L^{2}(\Omega)\right)^{n}: \operatorname{div} \mathbf{v} \in L^{2}(\Omega)\right\} \\
& H_{0, N}(\operatorname{div} ; \Omega)=\left\{\mathbf{v} \in H(\operatorname{div} ; \Omega): \mathbf{v} \cdot \mathbf{n}=0 \text { on } \Gamma_{N}\right\} .
\end{aligned}
$$

Remark 1 If $\Gamma_{N}$ is a subset of $\partial \Omega$ with positive measure, the space $H_{00}^{1 / 2}\left(\Gamma_{N}\right)$ consists of those elements $\xi \in H^{1 / 2}\left(\Gamma_{N}\right)$ whose trivial extension $\xi^{0}$ of $\xi$ by zero to all $\partial \Omega$ belongs to $H^{1 / 2}(\partial \Omega)[26]$, i.e.

$$
H_{00}^{1 / 2}\left(\Gamma_{N}\right)=\left\{\xi \in H^{1 / 2}\left(\Gamma_{N}\right) \mid \xi^{0} \in H^{1 / 2}(\partial \Omega)\right\}, \quad\|\xi\|_{H_{00}^{1 / 2}\left(\Gamma_{N}\right)}=\left\|\xi^{0}\right\|_{H^{1 / 2}(\partial \Omega)} .
$$


The condition $\mathbf{v} \cdot \mathbf{n}=0$ on $\Gamma_{N}$ means that $\left\langle\mathbf{v} \cdot \mathbf{n}, \xi^{0}\right\rangle_{\partial \Omega}=0$ for all $\xi \in$ $H_{00}^{1 / 2}\left(\Gamma_{N}\right)$, where $\langle\cdot, \cdot\rangle_{\partial \Omega}$ stands for the dual pairing. In the following we will use this notation: if $\mathbf{v} \in H_{0, N}(\operatorname{div} ; \Omega)$ then

$$
\int_{\Gamma_{D}} H_{D} \mathbf{v} \cdot \mathbf{n}:=\left\langle\mathbf{v} \cdot \mathbf{n}, \widetilde{H}_{D}\right\rangle_{\partial \Omega}
$$

being $\widetilde{H}_{D} \in H^{1 / 2}(\partial \Omega)$ any extension of $H_{D} \in H^{1 / 2}\left(\Gamma_{D}\right)$.

The variational formulation of (6) reads: find $(\mathbf{q}, p) \in H_{0, N}(\operatorname{div} ; \Omega) \times L^{2}(\Omega)$ such that

$$
\begin{aligned}
& \int_{\Omega}\left(\frac{\nu}{\kappa} \mathbf{q} \cdot \mathbf{v}-\operatorname{div} \mathbf{v} \frac{1}{\rho} p\right)=\int_{\Omega} \mathbf{f} \cdot \mathbf{v}-\int_{\Gamma_{D}} H_{D} \mathbf{v} \cdot \mathbf{n} \\
& \int_{\Omega} \operatorname{div} \mathbf{q} \frac{1}{\rho} \psi=0
\end{aligned}
$$

for all $(\mathbf{v}, \psi) \in H_{0, N}(\operatorname{div} ; \Omega) \times L^{2}(\Omega)$.

As usual, we consider the bilinear forms $a(\mathbf{w}, \mathbf{v})=\int_{\Omega} \frac{\nu}{\kappa} \mathbf{w} \cdot \mathbf{v}$ and $b(\mathbf{v}, \psi):=$ $\int_{\Omega} \operatorname{div} \mathbf{v} \frac{1}{\rho} \psi$ defined on $H_{0, N}(\operatorname{div} ; \Omega) \times H_{0, N}(\operatorname{div} ; \Omega)$ and $H_{0, N}(\operatorname{div} ; \Omega) \times L^{2}(\Omega)$ respectively. If $\Gamma_{D} \neq \emptyset$ it is easy to check that $a(\cdot, \cdot)$ is continuous and coercive on the space $V^{0}$ defined as

$$
\begin{aligned}
& V^{0}=\left\{\mathbf{v} \in H_{0, N}(\operatorname{div} ; \Omega): b(\mathbf{v}, \psi)=0 \forall \psi \in L^{2}(\Omega)\right\} \\
&=\left\{\mathbf{v} \in H_{0, N}(\operatorname{div} ; \Omega): \operatorname{div} \mathbf{v}=0\right\}
\end{aligned}
$$

and that the inf-sup condition is satisfied, namely such that

$$
\inf _{\psi \in Q} \sup _{\mathbf{v} \in V} \frac{b(\mathbf{v}, \psi)}{\|\mathbf{v}\|_{H(\operatorname{div} ; \Omega)}\|\psi\|_{L^{2}(\Omega)}}=: \beta>0,
$$

being $V=H_{0, N}(\operatorname{div} ; \Omega)$ and $Q=L^{2}(\Omega)$. If $\Gamma_{D}=\emptyset$ then the coercivity and inf-sup condition hold replacing $L^{2}(\Omega)$ by $L_{0}^{2}(\Omega)=\left\{\psi \in L^{2}(\Omega): \int_{\Omega} \psi=0\right\}$.

Remark 2 The mathematical tools introduced in this section are strictly related to those involved in the FEEC formulation of the previous section. The first two equations in (6) are the $L^{2}$ version of (1), with $s=0$. The functional spaces $L^{2}(\Omega), H(\operatorname{div} ; \Omega), H^{1}(\Omega)$ introduced above, are those of the Hilbert complex related with the de Rham sequences appearing on Fig.1. The variational formulation of (6) is given by, respectively, equations (4) and (5) in vector formalism, where $\partial \Omega=\Gamma_{D} \cup \Gamma_{N}$ together with $\operatorname{Tr}\left(*_{\frac{1}{\rho}} p\right)=H_{D}$ on $\Gamma_{D}$ and $\operatorname{Tr}(\mathbf{v})=0$ on $\Gamma_{N}$. Obtaining a well-posed numerical scheme for this problem requires to reproduce the de Rham sequence at the discrete level. This is detailed here below.

From now on, we assume that $\bar{\Omega}$ is covered by a simplicial mesh $\mathcal{T}$ with boundary faces located on either $\Gamma_{N}$ or $\Gamma_{D}$. For the sake of clarity we will assume that $\Gamma_{D} \neq \emptyset$. We consider the discretisation of the weak form of (6) involving Raviart-Thomas finite elements $R T_{r+1}$ of degree $r+1$ and discontinuous piecewise polynomial functions $D P_{r}$ of degree $r$, with $r \geq 0$. Let us recall 
that $R T_{r+1}(\mathcal{T})=\mathcal{P}_{r+1}^{-} \Lambda^{n-1}(\mathcal{T})$ and $D P_{r}(\mathcal{T})=\mathcal{P}_{r+1}^{-} \Lambda^{n}(\mathcal{T})$. Let us introduce the space $R T_{r+1}^{0, N}(\mathcal{T})=R T_{r+1}(\mathcal{T}) \cap H_{0, N}(\operatorname{div} ; \Omega)$, so that the discrete problem can be formulated as follows: find $\left(\mathbf{q}_{h}, p_{h}\right) \in R T_{r+1}^{0, N}(\mathcal{T}) \times D P_{r}(\mathcal{T})$ such that

$$
\begin{aligned}
& \int_{\Omega}\left(\frac{\nu}{\kappa} \mathbf{q}_{h} \cdot \mathbf{v}_{h}-\operatorname{div} \mathbf{v}_{h} \frac{1}{\rho} p_{h}\right)=\int_{\Omega} \mathbf{f} \cdot \mathbf{v}_{h}-\int_{\Gamma_{D}} H_{D} \mathbf{v}_{h} \cdot \mathbf{n}, \\
& \int_{\Omega} \operatorname{div} \mathbf{q}_{h} \frac{1}{\rho} \psi_{h}=0 .
\end{aligned}
$$

for all $\left(\mathbf{v}_{h}, \psi_{h}\right) \in R T_{r+1}^{0, N}(\mathcal{T}) \times D P_{r}(\mathcal{T})$.

It is easy to check that $a(\cdot, \cdot)$ is coercive in the space $V_{h}^{0}=\left\{\mathbf{v}_{h} \in R T_{r+1}^{0, N}(\mathcal{T})\right.$ : $\left.b\left(\mathbf{v}_{h}, \psi_{h}\right)=0 \forall \psi_{h} \in D P_{r}(\mathcal{T})\right\}$. In fact if $\mathbf{v}_{h} \in V_{h}^{0}$, taking $\psi_{h}=\operatorname{div} \mathbf{v}_{h} \in$ $D P_{r}(\mathcal{T})$ we have in particular $0=b\left(\mathbf{v}_{h}, \operatorname{div} \mathbf{v}_{h}\right)=\int_{\Omega} \frac{1}{\rho}\left(\operatorname{div} \mathbf{v}_{h}\right)^{2}$, hence

$$
V_{h}^{0}=\left\{\mathbf{v}_{h} \in R T_{r+1}^{0, N}(\mathcal{T}): \operatorname{div} \mathbf{v}_{h}=0\right\} \subset V^{0} .
$$

It is also well-known (see, e.g. [11]) that the discrete inf-sup condition is satisfied, namely,

$$
\inf _{\psi_{h} \in Q_{h}} \sup _{\mathbf{v}_{h} \in V_{h}} \frac{b\left(\mathbf{v}_{h}, \psi_{h}\right)}{\left\|\mathbf{v}_{h}\right\|_{H(\operatorname{div} ; \Omega)}\left\|\psi_{h}\right\|_{L^{2}(\Omega)}}=: \beta_{h}>0,
$$

being $Q_{h}=D P_{r}(\mathcal{T})$ and $V_{h}=R T_{r+1}^{0, N}(\mathcal{T})$. Moreover this condition holds uniformly with respect to h, i.e., $\beta_{h} \geq \beta_{0}>0$.

The matrix problem corresponding to the variational formulation (8) is of the following type

$$
\left[\begin{array}{cc}
A-B^{\top} \\
B & 0
\end{array}\right]\left[\begin{array}{l}
\mathbf{Q} \\
\mathbf{P}
\end{array}\right]=\left[\begin{array}{c}
\mathbf{F}-\mathbf{G} \\
\mathbf{0}
\end{array}\right]
$$

For given bases $\left\{\mathbf{w}_{h, j}\right\}_{j=1}^{\mathrm{N}_{0}}$ and $\left\{\pi_{h, i}\right\}_{i=1}^{\mathrm{M}}$ of $R T_{r+1}^{0, N}(\mathcal{T})$ and $D P_{r}(\mathcal{T})$, respectively (being $\mathrm{N}_{0}=\operatorname{dim} R T_{r+1}^{0, N}(\mathcal{T}) \leq \operatorname{dim} R T_{r+1}(\mathcal{T})=\mathrm{N}$ and $\mathrm{M}=\operatorname{dim} D P_{r}(\mathcal{T})$ ), the entries of the matrices $A \in \mathbb{R}^{\mathrm{N}_{0} \times \mathrm{N}_{0}}$ and $B \in \mathbb{R}^{\mathrm{M} \times \mathrm{N}_{0}}$ are

$$
A_{k, j}=a\left(\mathbf{w}_{h, j}, \mathbf{w}_{h, k}\right), \quad B_{i, j}=b\left(\mathbf{w}_{h, j}, \pi_{h, i}\right) .
$$

It is worth noting that the discrete inf-sup condition (9) is equivalent to $B$ being full rank. Similarly the components of the vectors $\mathbf{F}$ and $\mathbf{G} \in \mathbb{R}^{\mathrm{N}_{0}}$ are

$$
F_{k}=\int_{\Omega} \mathbf{f} \cdot \mathbf{w}_{h, k}, \quad G_{k}=\int_{\Gamma_{D}} H_{D} \mathbf{w}_{h, k} \cdot \mathbf{n} .
$$

The components of the vectors $\mathbf{Q} \in \mathbb{R}^{\mathrm{N}_{0}}$ and $\mathbf{P} \in \mathbb{R}^{\mathrm{M}}$ are the (unknown) coefficients of $\mathbf{q}_{h} \in R T_{r+1}^{0, N}(\mathcal{T})$ and $p_{h} \in D P_{r}(\mathcal{T})$ in the basis $\left\{\mathbf{w}_{h, j}\right\}_{j=1}^{\mathrm{N}_{0}}$ and $\left\{\pi_{h, i}\right\}_{i=1}^{\mathrm{M}}$, respectively. It is time to build up the bases $\left\{\mathbf{w}_{h, j}\right\}_{j=1}^{\mathrm{N}_{0}}$ and $\left\{\pi_{h, i}\right\}_{i=1}^{\mathrm{M}}$ for a suitable choice of unisolvent degrees of freedom for the fields in the spaces $R T_{r+1}^{0, N}(\mathcal{T})$ and $D P_{r}(\mathcal{T})$. This is the goal of the next section. 


\section{Weights on small simplices and bases in duality}

We are seeking for FE bases in duality with a set of degrees of freedom different from the classical moments, the weights on small simplices introduced in [29], that have a direct physical interpretation. These degrees of freedom for a $k$ form $w \in \mathcal{P}_{r+1}^{-} \Lambda^{k}(\mathcal{T})$ are integrals of $w$ on $k$-chains. This definition fits, for $r>0$, with the meaning of degrees of freedom as cochains, in the spirit of Whitney's original work.

We need to rely on multi-index notation $\boldsymbol{\alpha}=\left(\alpha_{0}, \ldots, \alpha_{n}\right) \in \mathbb{Z}_{+}^{n+1}$ and we define $|\boldsymbol{\alpha}|=\sum_{k=0}^{n} \alpha_{k}$. Then $\mathcal{I}_{r}^{n}=\left\{\boldsymbol{\alpha} \in \mathbb{Z}_{+}^{n+1}:|\boldsymbol{\alpha}|=r\right\}$. For any $\boldsymbol{\alpha} \in \mathcal{I}_{r}^{n}$ the small simplex $\{\boldsymbol{\alpha}, T\}$ is the $n$-simplex with barycentre at the point of coordinates $\sum_{i=0}^{n}\left[\left(\frac{1}{n+1}+\alpha_{i}\right) \mathbf{v}_{i}\right] /(r+1)$ and $1 /(r+1)$-homothetic to $T$. The latter expression signifies that $\{\boldsymbol{\alpha}, T\}$ has the same shape and orientation of $T$ and that its dimensions are reduced by a factor $1 /(r+1)$ with respect to those of $T$. For $k \in\{0,1, \ldots, n\}$ let us denote $\Delta_{k}(T)$ the set of $k$-subsimplices of $T$. For any $f \in \Delta_{k}(T)$, the $k$-simplex $\{\boldsymbol{\alpha}, f\}$ belongs to the small triangle $\{\boldsymbol{\alpha}, T\}$, it is parallel to $f$ and $1 /(r+1)$-homothetic to $f$. For the finite element approximation of Darcy problem we use the spaces $R T_{r+1}(\mathcal{T})=\mathcal{P}_{r+1}^{-} \Lambda^{n-1}(\mathcal{T})$ and $D P_{r}(\mathcal{T})=\mathcal{P}_{r+1}^{-} \Lambda^{n}(\mathcal{T})$.

Definition 1 For $r \geq 0$, in each $n$-simplex $T \in \mathcal{T}_{h}$, the weights of $\mathbf{v}_{h} \in R T_{r+1}$ are the functionals that associate $\mathbf{v}_{h}$ with its fluxes through the small $(n-1)$ faces of $T,\{\boldsymbol{\alpha}, f\}$, namely

$$
\xi_{\boldsymbol{\alpha}}^{f}: \mathbf{v}_{h} \mapsto \int_{\{\boldsymbol{\alpha}, f\}} \mathbf{v}_{h} \cdot \mathbf{n}_{k}^{T} \text { with } \boldsymbol{\alpha} \in \mathcal{I}_{r}^{n-1} \text { and } f \in \Delta_{n-1}(T),
$$

and the weights of $\psi_{h} \in D P_{r}$ are the functionals that associate $\psi_{h}$ with its integrals over the small $n$-simplices $\{\boldsymbol{\beta}, T\}$, namely

$$
\xi_{\boldsymbol{\beta}}^{T}: \psi_{h} \mapsto \int_{\{\boldsymbol{\beta}, T\}} \psi_{h} \text { with } \boldsymbol{\beta} \in \mathcal{I}_{r}^{n}
$$

In general the weights of elements of $\mathcal{P}_{r+1}^{-} \Lambda^{k}(\mathcal{T})$ are integrals on the small $k$-simplices, the so called $k$-weights. In [16] it has been proved that these degrees of freedom are unisolvent. However it is well know that the number of small $n$-simplices is equal to the dimension of $\mathcal{P}_{r+1}^{-} \Lambda^{n}(\mathcal{T})$ but the number of small $(n-1)$-simplices is bigger than the dimension of $\mathcal{P}_{r+1}^{-} \Lambda^{n-1}(\mathcal{T})$. In [2] a minimal set of unisolvent $k$-weights is identified. In the particular case of $k=n-1$ a minimal set of $(n-1)$-weights is that obtained by eliminating those weights associated with the small $(n-1)$-faces at the interior of any element $T \in \mathcal{T}$ that are parallel to one of the faces of $T$, for instance, parallel to the face opposite to the first vertex of $T$.

The spaces $R T_{r+1}(\mathcal{T})$ and $D P_{r}(\mathcal{T})$ are constructed locally, on one element $T \in \mathcal{T}$, and then local contributions are assembled by relying on local-to-global numbering of the degrees of freedom, as it is usual in finite elements. The 
dimensions of the spaces $R T_{r+1}(T)$ and $D P_{r}(T)$ (see e.g. [28], with $\mathbf{x} \in \mathbb{R}^{n}$ ) are

$$
\begin{gathered}
\mathbb{N}_{\text {loc }}=\operatorname{dim} R T_{r+1}(T)=\operatorname{dim}\left(\mathbb{P}_{r}^{n} \oplus \mathbf{x} \mathbb{P}_{r}\right)=\frac{(n+r-1) !(n+r+1)}{(n-1) ! r !}, \\
\mathrm{M}_{1 \mathrm{oc}}=\operatorname{dim} D P_{r}(T)=\operatorname{dim} \mathbb{P}_{r}=\frac{(n+r) !}{n ! r !} .
\end{gathered}
$$

We now introduce a basis $\left\{\mathbf{w}_{h, j}\right\}_{j=1}^{\mathrm{N}_{10 \mathrm{c}}} \subset R T_{r+1}(T)$ and a basis $\left\{\pi_{h, i}\right\}_{i=1}^{\mathrm{M}_{10 \mathrm{c}}} \subset$ $D P_{r}(T)$, that are in duality with suitable degrees of freedom for $\mathbf{v}_{h \mid T} \in$ $R T_{r+1}(T)$ and $q_{h \mid T} \in D P_{r}(T)$, respectively. We recall that, given a basis $\left\{\phi_{j}\right\}_{j}$ of a finite-dimensional vector space $P$ and a set $\left\{\sigma_{i}\right\}_{i}$ of the dual space $P^{\prime}$, we say that $\left\{\phi_{j}\right\}_{j}$ is in duality with $\left\{\sigma_{i}\right\}_{i}$ if $\sigma_{i}\left(\phi_{j}\right)=\delta_{i, j}$, being $\delta$.. the Kronecker's symbol. Starting from a generic basis $\left\{\psi_{j}\right\}_{j}$ of $P$, we can build up the basis $\left\{\phi_{j}\right\}_{j}$ in duality with the selected functionals $\left\{\sigma_{i}\right\}_{i}$, by solving a linear system with matrix $V_{i j}=\sigma_{i}\left(\psi_{j}\right)$, known in the literature as generalised Vandermonde matrix. For $r>0$, the definition of FE bases $\left\{\mathbf{w}_{h, j}\right\}_{j=1}^{\mathbb{N}_{10 c}} \subset R T_{r+1}(T)$ (resp. $\left.\left\{\pi_{h, i}\right\}_{i=1}^{\mathrm{M}_{10 \mathrm{c}}} \subset D P_{r}(T)\right)$ in duality with suitable unisolvent degrees of freedom $\left\{\sigma_{i}\right\}_{i}$ involves the multiplication by the inverse of the corresponding generalised Vandermonde matrix, as explained in [13]. We now detail the construction of the FE bases in duality with the weights on the small simplices.

The generalised Vandermonde matrix for an element $T \in \mathcal{T}$ is computed from a known basis of the polynomial space associated with $\mathcal{P}_{r+1}^{-} \Lambda^{k}(T)$, for instance, a basis with elements of the form $\lambda^{\boldsymbol{\alpha}} \omega_{f}$ with $\boldsymbol{\alpha} \in \mathcal{I}_{r}^{n}$ and $f \in$ $\Delta_{k}(T)$, being $\omega_{f} \in \mathcal{P}_{1}^{-} \Lambda^{k}(T)$ the differential Whitney $k$-form of polynomial degree 1 associated with the $k$-subsimplex $f$ (see [29]). There is a natural correspondence between these polynomial $k$-forms and the $k$-simplices $\{\boldsymbol{\alpha}, f\}$. The set $\left\{\lambda^{\boldsymbol{\alpha}} \omega_{f}: \boldsymbol{\alpha} \in \mathcal{I}_{r}^{n-1}, f \in \Delta_{n-1}(T)\right\}$ generates $\mathcal{P}_{r+1}^{-} \Lambda^{n-1}(T)$ but it is clear that it is not a basis because, as mentioned before, the number of small $(n-1)$-simplices is bigger than the dimension of $\mathcal{P}_{r+1}^{-} \Lambda^{n-1}(T)$. It is well known (see, e.g., [9]) that to obtain a basis it is enough to eliminate those elements with $f=T \backslash \mathbf{v}_{0}^{T}$, being $\mathbf{v}_{0}^{T}$ the first vertex of $T$, namely the vertex of $T$ which carries the smallest number, among the $n+1$ local vertices of $T$, in the global numbering of the mesh nodes, and $\alpha_{0} \neq 0$. Notice that this selection criterium corresponds to the one adopted to define a minimal set of unisolvent weights. In the following, we will just write $\mathbf{v}_{0}$ instead of $\mathbf{v}_{0}^{T}$. On a generic $n$-simplex $T \in \mathcal{T}$, the generalised Vandermonde matrix for the Raviart-Thomas finite elements is a square matrix of size $\mathrm{N}_{10 c}$ and has entries

$$
\left(V_{i j}^{R T}=\right) \quad V_{(\boldsymbol{\alpha}, f),\left(\boldsymbol{\alpha}^{\prime}, f^{\prime}\right)}^{R T}=\int_{\{\boldsymbol{\alpha}, f\}} \lambda^{\boldsymbol{\alpha}^{\prime}} \omega_{f^{\prime}} \cdot \mathbf{n}_{f} .
$$

Here $\boldsymbol{\alpha}, \boldsymbol{\alpha}^{\prime} \in \mathcal{I}_{r}^{n-1}$, the subsimplices $f, f^{\prime} \in \Delta_{n-1}(T)$, and $\alpha_{0}=0$ if $f=T \backslash \mathbf{v}_{0}$ and $\alpha_{0}^{\prime}=0$ if $f^{\prime}=T \backslash \mathbf{v}_{0}$. For the discontinuous piecewise polynomial finite elements, the generalised Vandermonde matrix is square, of size $M_{10 c}$ and has entries

$$
\left(V_{\ell m}^{D P}=\right) \quad V_{(\boldsymbol{\alpha}, T),\left(\boldsymbol{\alpha}^{\prime}, T\right)}^{D P}=\int_{\{\boldsymbol{\alpha}, T\}} \lambda^{\boldsymbol{\alpha}^{\prime}},
$$

with $\boldsymbol{\alpha}, \boldsymbol{\alpha}^{\prime} \in \mathcal{I}_{r}^{n}$. 
The inverse matrix $\left(V^{R T}\right)^{-1}$ (resp. $\left.\left(V^{D P}\right)^{-1}\right)$ gives, column by column, the coefficients to express the functions $\left\{\mathbf{w}_{h, j}\right\}_{j=1}^{\mathrm{N}_{\text {loc }}}$ (resp. $\left\{\pi_{h, \ell}\right\}_{\ell=1}^{\mathrm{M}_{\text {loc }}}$ ) of the dual basis of $R T_{r+1}(T)$ (resp. of $D P_{r}(T)$ ) as a linear combination of the basis $\left\{\mathbf{y}_{h, j}\right\}_{j=1}^{N_{10 c}}$ (resp. $\left\{z_{h, \ell}\right\}_{\ell=1}^{M_{\text {loc }}}$ ) with elements of the form $\mathbf{y}_{h, j}=\lambda^{\boldsymbol{\alpha}_{\omega_{f}}}$ (resp. $\left.z_{h, \ell}=\lambda^{\boldsymbol{\alpha}} \omega_{T}\right)$ that is

$\mathbf{w}_{h, j}(\mathbf{x})=\sum_{i=1}^{\mathrm{N}_{\mathrm{loc}}}\left[\left(V^{R T}\right)^{-1}\right]_{i, j} \mathbf{y}_{h, i}(\mathbf{x}) \quad\left(\pi_{h, \ell}(\mathbf{x})=\sum_{s=1}^{\mathrm{M}_{\mathrm{1oc}}}\left[\left(V^{D P}\right)^{-1}\right]_{s, \ell} z_{h, s}(\mathbf{x})\right)$.

If $\mathbf{Q}$ is the vector with entries the weights of a discrete function $\mathbf{q}_{h \mid T}$ and $\left\{\mathbf{w}_{h, j}\right\}_{j=1}^{N_{1 \circ c}} \subset R T_{r+1}(T)$ is the basis in duality with the set of weights, one has

$$
\begin{aligned}
\mathbf{q}_{h \mid T}(\mathbf{x}) & =\sum_{j=1}^{\mathrm{N}_{\text {loc }}} Q_{j} \mathbf{w}_{h, j}(\mathbf{x})=\sum_{j=1}^{\mathrm{N}_{\text {loc }}} Q_{j} \sum_{i=1}^{\mathrm{N}_{\text {loc }}}\left[\left(V^{R T}\right)^{-1}\right]_{i, j} \mathbf{y}_{h, i}(\mathbf{x}) \\
& =\sum_{i=1}^{\mathrm{N}_{\text {loc }}} \sum_{j=1}^{\mathrm{N}_{\text {loc }}}\left[\left(V^{R T}\right)^{-1}\right]_{i, j} Q_{j} \mathbf{y}_{h, i}(\mathbf{x})=\sum_{i=1}^{\mathrm{N}_{\text {loc }}}\left(\mathbf{Q}^{T} \cdot\left[\left(V^{R T}\right)^{-1}\right]_{i, \cdot}\right) \mathbf{y}_{h, i}(\mathbf{x})
\end{aligned}
$$

In [13], it has been stated that the independence of a degree of freedom from the metric of the subsimplex of $T$ that supports it makes the entries of the Vandermonde matrix, such as $V^{R T}$ or $V^{D P}$, computable once on a generic triangle $T$ and valid in any other triangle $T^{\prime}$ different from $T$, up to a suitable renumbering of the indices. The inverse matrix $\left(V^{R T}\right)^{-1}$ (resp. $\left.\left(V^{D P}\right)^{-1}\right)$ of size $\mathrm{N}_{\text {loc }} \ll N_{0}$ (resp. $\left.\mathrm{M}_{\text {loc }} \ll M\right)$ is thus computed only once for a generic $T$ but it is valid for all $T \in \mathcal{T}$, up to a suitable renumbering of the indices. The value $\mathbf{q}_{h}(\mathbf{x})$ of the discrete field $\mathbf{q}_{h}$ at $\mathbf{x} \in \Omega$ is computed as $\mathbf{q}_{h}(\mathbf{x})=\sum_{T \in \mathcal{T}}^{\prime} \mathbf{q}_{h \mid T}(\mathbf{x})$ where $\sum^{\prime}$ stands for assembling the local contributions $\mathbf{q}_{h \mid T}(\mathbf{x})$ associated with the elements $T \in \mathcal{T}$.

\section{Numerical evaluation of the inf-sup condition}

Before solving the Darcy's problem, we wish to estimate numerically the constant of the inf-sup condition, using the matrices computed with the bases defined in the previous section. This is a first important difficulty to face: we thus check that the new proposed FE approach is stable and that the computed quantity (the inf-sup constant) converges to the analytic one (a known value stated by theoretical results), for $h$ decreasing or $r$ increasing.

Coming back to the linear system (10), it is easy to check that $A$ is invertible. It is in fact symmetric definite positive. The vector $\mathbf{Q}$ can be eliminated yielding

$$
S \mathbf{P}=-B A^{-1}(\mathbf{F}-\mathbf{G}) \text { being } S=B A^{-1} B^{T} .
$$

The matrix $S$ is called the Schur complement of $A$. Let us denote $M \in \mathbb{R}^{\mathrm{M} \times \mathrm{M}}$ the matrix with entries $M_{i, j}=\int_{\Omega} \pi_{h, j} \pi_{h, i}$, We recall the following result (see the proof in [20]). 
Proposition 1 Assume that the bilinear form $a(\cdot, \cdot)$ is symmetric continuous with constants $C_{a}$ and coercive on $V_{h}$ with constants $\widetilde{\alpha}$ and that the bilinear form $b(\cdot, \cdot)$ is continuous with constants $C_{b}$ and that the inf-sup condition (9) holds. Then the matrices $S$ and $M$ are spectrally equivalent, i.e., the following holds for all $\boldsymbol{\Phi} \in \mathbb{R}^{\mathrm{M}}$

$$
\frac{\beta_{h}^{2}}{C_{a}} \leq \frac{\boldsymbol{\Phi}^{T} S \boldsymbol{\Phi}}{\boldsymbol{\Phi}^{T} M \boldsymbol{\Phi}} \leq \frac{C_{b}^{2}}{\widetilde{\alpha}}
$$

Notice that if we set the physical parameters equal to one then we have $C_{a}=C_{b}=1$. We have:

$$
\operatorname{Sup}_{\mathbf{v}_{h} \in R T_{r+1}^{0, N}} \frac{\int_{\Omega} \operatorname{div} \mathbf{v}_{h} \psi_{h}}{\left\|\mathbf{v}_{h}\right\|_{\left(L^{2}(\Omega)\right)^{n}}}=\operatorname{Sup}_{\mathbf{V} \in \mathbb{R}^{\mathrm{N}_{0}}} \frac{\boldsymbol{\Phi}^{T} B \mathbf{V}}{\left(\mathbf{V}^{T} A \mathbf{V}\right)^{1 / 2}}
$$

Taking in particular $\mathbf{V}=A^{-1} B^{T} \boldsymbol{\Phi}$ and recalling that $S=B A^{-1} B^{T}$ one has

$$
\operatorname{Sup}_{\mathbf{V} \in \mathbb{R}^{\mathbb{N}_{0}}} \frac{\boldsymbol{\Phi}^{T} B \mathbf{V}}{\left(\mathbf{V}^{T} A \mathbf{V}\right)^{1 / 2}} \geq \frac{\boldsymbol{\Phi}^{T} S \boldsymbol{\Phi}}{\left(\boldsymbol{\Phi}^{T} S \boldsymbol{\Phi}\right)^{1 / 2}}=\left(\boldsymbol{\Phi}^{T} S \boldsymbol{\Phi}\right)^{1 / 2}
$$

Let $\lambda_{*}$ and $\lambda^{*}$ be respectively the smallest and the biggest eigenvalues of the following generalised problem: find a scalar $\lambda$ and a vector $\boldsymbol{\Phi} \neq \mathbf{0}$ such that

$$
S \boldsymbol{\Phi}=\lambda M \Phi .
$$

Then

$$
\lambda_{*}=\operatorname{Inf}_{\boldsymbol{\Phi} \in \mathbb{R}^{M}} \frac{\boldsymbol{\Phi}^{T} S \boldsymbol{\Phi}}{\boldsymbol{\Phi}^{T} M \boldsymbol{\Phi}} \text { and } \lambda^{*}=\operatorname{Sup}_{\boldsymbol{\Phi} \in \mathbb{R}^{\mathrm{M}}} \frac{\boldsymbol{\Phi}^{T} S \boldsymbol{\Phi}}{\boldsymbol{\Phi}^{T} M \boldsymbol{\Phi}}
$$

Hence

$$
\beta_{h}^{2}=\lambda_{*} \leq \frac{\boldsymbol{\Phi}^{T} S \boldsymbol{\Phi}}{\boldsymbol{\Phi}^{T} M \boldsymbol{\Phi}} \leq \lambda^{*}=\frac{1}{\widetilde{\alpha}} .
$$

So we can estimate $\beta_{h}$ by computing the smallest eigenvalue of the generalised eigenvalue problem (14) (see [21]; see also [28], [12]).

Let us consider the infinite-dimensional counterpart. If $\Gamma_{D} \neq \emptyset$ then for any $\psi \in L^{2}(\Omega)$ there exists a unique $u_{\psi} \in H_{0, \Gamma_{D}}^{1}(\Omega):=\left\{\zeta \in H^{1}(\Omega): \gamma \xi_{\mid \Gamma_{D}}=0\right\}$ such that

$$
\int_{\Omega} \operatorname{grad} u_{\psi} \cdot \operatorname{grad} \xi=-\int_{\Omega} \psi \xi, \quad \forall \xi \in H_{0, \Gamma_{D}}^{1}(\Omega) .
$$

Let us denote $C_{\Omega}$ the constant in the Poincare's inequality

$$
\|\xi\|_{L^{2}(\Omega)} \leq C_{\Omega}\|\operatorname{grad} \xi\|_{\left(L^{2}(\Omega)\right)^{n}}, \quad \forall \xi \in H_{0, \Gamma_{D}}^{1}(\Omega)
$$

Clearly

$$
\left\|\operatorname{grad} u_{\psi}\right\|_{\left(L^{2}(\Omega)\right)^{n}}^{2} \leq\|\psi\|_{L^{2}(\Omega)}\left\|u_{\psi}\right\|_{L^{2}(\Omega)} \leq\|\psi\|_{L^{2}(\Omega)} C_{\Omega}\left\|\operatorname{grad} u_{\psi}\right\|_{\left(L^{2}(\Omega)\right)^{n}}
$$




\begin{tabular}{|c|cccccc|}
\hline \multicolumn{1}{|c|}{$h=1 / J$} \\
$r+1$ & $1 / 4$ & $1 / 6$ & $1 / 8$ & $1 / 10$ & $1 / 20$ & $1 / 30$ \\
\hline 1 & 4.478674 & 4.459351 & 4.452257 & 4.448915 & 4.444339 & 4.443977 \\
2 & 4.444860 & 4.443288 & 4.443012 & 4.442936 & 4.442936 & 4.442936 \\
3 & 4.442914 & 4.442886 & 4.442883 & 4.442883 & 4.442883 & 4.442883 \\
4 & 4.442883 & 4.442883 & 4.442883 & 4.442883 & 4.442883 & 4.442883 \\
\hline
\end{tabular}

Table 1 Inf-sup constant $\beta_{h}$ varying the polynomial approximation degree $r$ and the mesh size $h$ over the domain $\bar{\Omega}=[0,1]^{2}$ with $\Gamma_{D}=\partial \Omega$.

\begin{tabular}{|c|cccccc|}
\hline \multicolumn{1}{|c|}{$h=1 / J$} \\
$r+1$ & $1 / 4$ & $1 / 6$ & $1 / 8$ & $1 / 10$ & $1 / 20$ & $1 / 30$ \\
\hline 1 & 3.114585 & 3.129624 & 3.134863 & 3.137286 & 3.140516 & 3.141114 \\
2 & 3.141637 & 3.141601 & 3.141595 & 3.141594 & 3.141593 & 3.141593 \\
3 & 3.141593 & 3.141593 & 3.141593 & 3.141593 & 3.141593 & 3.141593 \\
4 & 3.141593 & 3.141593 & 3.141593 & 3.141593 & 3.141593 & 3.141593 \\
\hline
\end{tabular}

Table 2 Inf-sup constant $\widetilde{\beta}_{h}$ varying the polynomial approximation degree $r$ and the mesh size $h$ over the domain $\bar{\Omega}=[0,1]^{2}$ with $\Gamma_{D} \neq \emptyset$ and $\Gamma_{N} \neq \emptyset$.
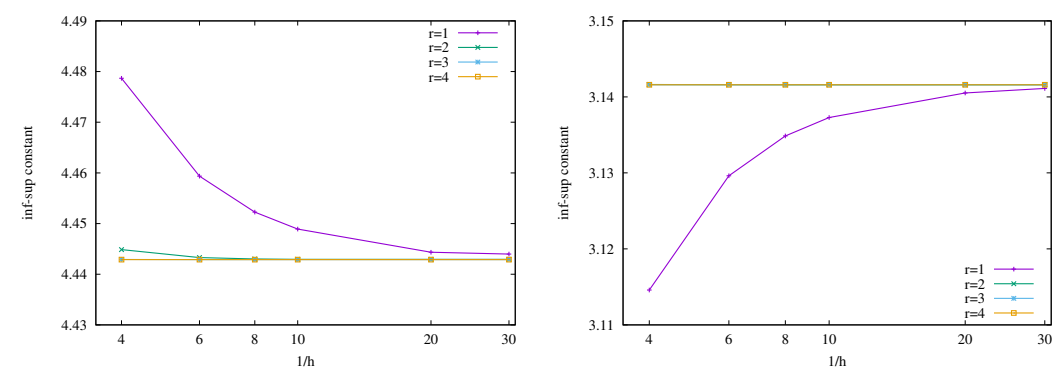

Fig. 2 Convergence of the inf-sup constant $\widetilde{\beta}_{h}$ to the theoretical value $\sqrt{2} \pi$ (left) and $\pi$ (right) for $h \rightarrow 0$, varying the approximation degree $r$ (see Tables 1 and 2, resp., for the computed values of $\widetilde{\beta}_{h}$ ).

hence $\left\|\operatorname{grad} u_{\psi}\right\|_{\left(L^{2}(\Omega)\right)^{n}} \leq C_{\Omega}\|\psi\|_{L^{2}(\Omega)}$. Denoting $\mathbf{z}_{\psi}=\operatorname{grad} u_{\psi}$, since we have $\operatorname{div} \mathbf{z}_{\psi}=\psi$ one gets

$$
\begin{aligned}
& \operatorname{Sup}_{\mathbf{v} \in H_{0, N}}(\operatorname{div} ; \Omega) \frac{\int_{\Omega} \operatorname{div} \mathbf{v} \psi}{\|\mathbf{v}\|_{\left(L^{2}(\Omega)\right)^{n}}} \geq \frac{\int_{\Omega} \operatorname{div} \mathbf{z}_{\psi} \psi}{\left\|\mathbf{z}_{\psi}\right\|_{\left(L^{2}(\Omega)\right)^{n}}} \\
& \quad=\frac{\|\psi\|_{L^{2}(\Omega)}^{2}}{\left\|\operatorname{grad} u_{\psi}\right\|_{\left(L^{2}(\Omega)\right)^{n}}} \geq \frac{1}{C_{\Omega}}\|\psi\|_{L^{2}(\Omega)} .
\end{aligned}
$$

Table 1 shows the square of the smallest eigenvalue of (14) set in the domain $\bar{\Omega}=[0,1]^{2}$ with $\Gamma_{D}=\partial \Omega$, varying the polynomial approximation degree $r$ and the mesh size $h$. It converges to $\sqrt{2} \pi \approx 4.442883$ that is the infinite-dimensional counterpart. In fact, it is known (see, e.g., [25]) that if $\Omega=(0, a) \times(0, b) \subset \mathbb{R}^{2}$ and $\Gamma_{D}=\partial \Omega$ then the sharp constant in (15) is $C_{\Omega}=\sigma_{D}^{-1 / 2}$ being $\sigma_{D}=(\pi / a)^{2}+(\pi / b)^{2}$ the smallest positive eigenvalue of the Dirichlet Laplacian.

Table 2 shows the square of the smallest eigenvalue of (14) set in the domain $\bar{\Omega}=[0,1]^{2}$ with $\Gamma_{D}$ equal to the vertical sides of $\Omega$ and $\Gamma_{N}$ equal to 
the horizontal ones, varying the polynomial approximation degree $r$ and the mesh size $h$. Figure 2 shows the convergence of the inf-sup constant $\beta_{h}$ to the theoretical value $\pi$ for $h \rightarrow 0$, varying the approximation degree $r$. For $r=0$ a rather fine mesh is necessary to have $\beta_{h}$ close to $\pi$. As soon as $r>0$, the value $\beta_{h}$ is a good approximation of $\pi$ already on a very coarse mesh.

\section{Solving the indefinite linear system}

A second difficulty to face with the considered Darcy's problem is the imposition of the divergence free constraint on the fluid discharge. We propose a new technique for solving the linear system (10). It is inspired on the tree-cotree decomposition of the unknowns, presented, for low order Raviart-Thomas FEs, in [7]. According to [6], using the weights as degrees of freedom for fields in the spaces $R T_{r+1}(\mathcal{T})$ and $D P_{r}(\mathcal{T})$, with $r \geq 0$, the matrix associated with the divergence operator $D$ is the incidence matrix of a directed graph $\mathcal{G}^{*}=\left(\mathcal{V}^{*}, \mathcal{E}^{*}\right)$. This fact yields a generalisation to the high-order case $r>0$ of a procedure analogous to the one proposed in [7] for $r=0$. By choosing a spanning tree of this graph, it is possible to identify an invertible square submatrix of the divergence matrix. Then, by reordering the unknowns associated with the arcs of the tree and of the cotree, a solution of the linear system (10) can be found using a simple elimination procedure. The same decomposition can be applied when using the classical moments as degrees of freedom (see [4]). Note that moments and weights coincide for $r=0$. When $r>0$, adopting the weights on the small simplices as degrees of freedom, the tree-cotree decomposition turns out to be more natural.

Remark 3 This is related with the construction of Raviart-Thomas divergencefree basis. The problem for elements of degree one has been studied in different papers (see, e.g., [23], [19], [32], [30], [5]). For Raviart-Thomas finite elements of higher degree, the problem has been studied in [3] and [4] using moments as degrees of freedom. Here, we test high order Raviart-Thomas with weights as degrees of freedom, which makes the construction of the matrix of the divergence operator quite simple.

The graph $\mathcal{G}^{*}=\left(\mathcal{V}^{*}, \mathcal{E}^{*}\right)$ has a node for each small $n$-simplex plus an additional node corresponding to the exterior of the domain, and an arc for each small $(n-1)$-simplex associated to the minimal set of $(n-1)$-weights. In Fig. 3, we show the arcs of the graph $\mathcal{G}^{*}$ for $n=2$ on a partial configuration with three elements $[0,1,2],[0,2,3],[1,2,4]$ selected in the mesh, each of them having one side on $\partial \Omega$, and an example of spanning tree. When using a basis in duality with the weights, the divergence operator $D$ is the incidence matrix of $\mathcal{G}^{*}$ with root the node $\mathbf{v}_{\text {ext }}$ corresponding to the exterior of the domain.

It is well-known that the submatrix of an incidence matrix of a connected directed graph corresponding to a spanning tree is invertible [33]. In particular if one decomposes the divergence matrix as $D=\left[D_{s t}, D_{c t}\right]$ being $D_{s t}$ the submatrix of $D$ with columns corresponding to edges in $\mathcal{F}^{*}$ (the spanning 

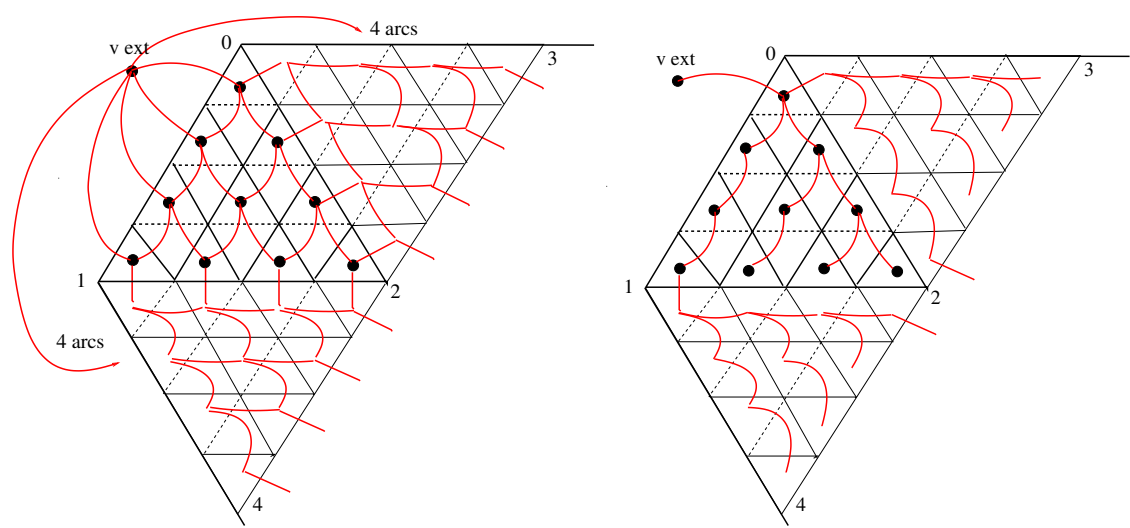

Fig. 3 An example of a primal graph (left) and of a spanning tree (right) on the small mesh of three elements $[0,1,2],[1,2,4],[0,2,3]$ of $\mathcal{T}_{h}$, with edges $[0,1],[1,4],[0,3] \in \partial \Omega$, whereas $[1,2]$ is also on the boundary of $[1,2,4]$ and $[0,2]$ on that of $[0,2,3]$. The doted arcs are not on the graph because they are associated to weights that are not in the minimal set.

tree), and $D_{c t}$ the submatrix with the columns left (the cotree), then $D_{s t}$ is invertible. This can be extended also to the matrix $B$ of system (10) which is defined as

$$
B_{i, j}=b\left(\mathbf{w}_{h, j}, \pi_{h, i}\right) .
$$

Notice that the set $\left\{\mathbf{w}_{h, j}\right\}_{j=1}^{\mathrm{N}_{0}}$ does not include the basis functions corresponding to the small edges on $\Gamma_{N}$. Using the same decomposition as for $D$, one gets

$$
B=\left[B_{s t}, B_{c t}\right]
$$

Lemma $1 B_{\text {st }}$ is invertible.

Proof Let $\mathbf{V}_{s t}$ be such that $B_{s t} \mathbf{V}_{s t}=\mathbf{0}$. Then, taking

$$
\mathbf{V}=\left[\begin{array}{c}
\mathbf{V}_{s t} \\
\mathbf{0}
\end{array}\right]
$$

follows that $B \mathbf{V}=\mathbf{0}$. This means that $\operatorname{div} \mathbf{v}_{h}=0$ being $\mathbf{v}_{h}$ the element of $R T_{r+1}^{0, N}$ with weights given by the coefficients of $\mathbf{V}$. Then $D \mathbf{V}=\mathbf{0}$ and in particular, $D_{s t} \mathbf{V}_{s t}=\mathbf{0}$. Since $D_{s t}$ is non-singular it follows that $\mathbf{V}_{s t}=\mathbf{0}$. Thus $B_{s t}$ is non-singular.

Similarly, one can decompose the matrix $A$ as follows

$$
A=\left[\begin{array}{ll}
A_{s t, s t} & A_{s t, c t} \\
A_{c t, s t} & A_{c t, c t}
\end{array}\right] .
$$

Thus, we have an indefinite linear system equivalent to (10), namely

$$
\left[\begin{array}{ccc}
A_{s t, s t} & A_{s t, c t} & -B_{s t}^{\top} \\
A_{c t, s t} & A_{c t, c t}-B_{c t}^{\top} \\
B_{s t} & B_{c t} & 0
\end{array}\right]\left[\begin{array}{c}
\mathbf{Q}_{s t} \\
\mathbf{Q}_{c t} \\
\mathbf{P}
\end{array}\right]=\left[\begin{array}{c}
\mathbf{F}_{s t}-\mathbf{G}_{s t} \\
\mathbf{F}_{c t}-\mathbf{G}_{c t} \\
\mathbf{0}
\end{array}\right]
$$


It remains only to eliminate $\mathbf{Q}_{s t}$ and $\mathbf{P}$ in the following way

$$
\begin{gathered}
\mathbf{Q}_{s t}=-B_{s t}^{-1} B_{c t} \mathbf{Q}_{c t}, \\
\mathbf{P}=-B_{s t}^{-\top}\left(\mathbf{F}_{s t}-\mathbf{G}_{s t}-A_{s t, c t} \mathbf{Q}_{c t}+A_{s t, s t} B_{s t}^{-1} B_{c t} \mathbf{Q}_{c t}\right) .
\end{gathered}
$$

Then, the system to solve is

$$
\tilde{A} \mathbf{Q}_{c t}=\tilde{\mathbf{F}},
$$

where

$$
\begin{gathered}
\tilde{A}=A_{c t, c t}-A_{c t, s t} B_{s t}^{-1} B_{c t}+B_{c t}^{\top} B_{s t}^{-\top}\left[-A_{s t, c t}+A_{s t, s t} B_{s t}^{-1} B_{c t}\right] \\
\tilde{\mathbf{F}}=\mathbf{F}_{c t}-\mathbf{G}_{c t}+B_{s t}^{-\top}\left(\mathbf{F}_{s t}-\mathbf{G}_{s t}\right) .
\end{gathered}
$$

Note that if $A_{s t, c t}^{\top}=A_{c t, s t}$, then $\tilde{A}$ is symmetric since we can write

$$
\tilde{A}=A_{c t, c t}+\left(B_{s t}^{-1} B_{c t}\right)^{\top} A_{s t, s t}\left(B_{s t}^{-1} B_{c t}\right)-A_{c t, s t} B_{s t}^{-1} B_{c t}-\left(A_{s t, c t}^{\top} B_{s t}^{-1} B_{c t}\right)^{\top} .
$$

\section{Numerical results}

Numerical tests are done for the Darcy's problem (6) in the particular case of a two dimensional domain and a homogeneous medium with $\nu / \kappa=1$ and $\rho=1$. We have considered a square domain $\Omega=[0,1]^{2}$ and assumed that the flow across the bottom and top of $\Omega$, is zero, namely, $\mathbf{q} \cdot \mathbf{n}=0$ on $\Gamma_{N}=$ $(0,1) \times\{0,1\}$. (Note that in the two-dimensional problem, $x_{2}$ is the elevation.) On $\Gamma_{D}=\{0,1\} \times[0,1]$ we imposed $p=H_{D}$ being

$$
H_{D}(\mathbf{x})=\left\{\begin{array}{l}
1 \text { if } x_{1}=0 \\
0 \text { if } x_{1}=1
\end{array}\right.
$$

In order to check the convergence rate we construct a synthetic problem with pressure $p_{e x}=1-x_{1}$ (that clearly verifies the boundary condition on $\Gamma_{D}$ ), and discharge (see a visualisation in Fig. 4)

$$
\mathbf{q}_{e x}=\left(\begin{array}{c}
\frac{\pi}{4} x_{1}^{2} x_{2} \sin \left(\frac{\pi}{2} x_{2}\right)-\frac{1}{2} x_{1}^{2} \cos \left(\frac{\pi}{2} x_{2}\right) \\
x_{1} x_{2} \cos \left(\frac{\pi}{2} x_{2}\right)
\end{array}\right)
$$

that verifies the boundary conditions on $\Gamma_{N}$ and is divergence-free.

For the finite element approximation of the discharge and the pressure we use the space $R T_{r+1}^{0, N}(\mathcal{T})$ and $D P_{r}(\mathcal{T})$ respectively with $r=\{0,1,2,3\}$ over successive uniformly refined oriented meshes $\mathcal{T}$ of the domain $\Omega$. The meshes $\mathcal{T}$ are constructed by dividing $\Omega$ into $J^{2}, J=2, \ldots, 10$, rectangles and then, each of them, in two triangles, to finally get $2 J^{2}$ (big) triangles. We use bases in duality with the weights on the small simplices that are associated with the principal lattice of order $r$ in each element of the mesh.

Numerical experiments consist in computing the discrete solution solving the linear systems (10) and (20) by using the direct solver of Matlab. The 

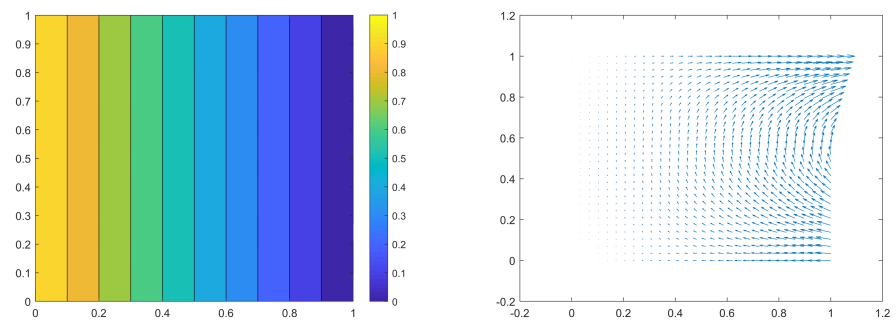

Fig. 4 Computed pressure (left) and discharge (right) for the considered synthetic Darcy's problem in $\bar{\Omega}=[0,1]^{2}$ with physical parameters $\nu / k=1, \rho=1$.

\begin{tabular}{|c|c|c|c|c|}
\hline \multirow{2}{*}{$r+1$} & \multicolumn{4}{|c|}{$h=1 / J$} \\
\cline { 1 - 1 } & $1 / 4$ & $1 / 6$ & $1 / 8$ & $1 / 10$ \\
\hline 2 & $1.582 \mathrm{e}-01$ & $1.115 \mathrm{e}-01$ & $8.469 \mathrm{e}-02$ & $6.797 \mathrm{e}-02$ \\
3 & $1.169 \mathrm{e}-02$ & $5.295 \mathrm{e}-03$ & $3.006 \mathrm{e}-03$ & $1.934 \mathrm{e}-03$ \\
4 & $7.101 \mathrm{e}-04$ & $2.395 \mathrm{e}-04$ & $1.090 \mathrm{e}-04$ & $5.813 \mathrm{e}-05$ \\
& $3.043 \mathrm{e}-05$ & $6.086 \mathrm{e}-06$ & $1.938 \mathrm{e}-06$ & $7.985 \mathrm{e}-07$ \\
\hline
\end{tabular}

\begin{tabular}{|c|c|c|c|c|}
\hline \multirow{3}{*}{$r+1$} & \multicolumn{4}{|c|}{$h=1 / J$} \\
\cline { 1 - 1 } 2 & $1 / 4$ & $1 / 6$ & $1 / 8$ & $1 / 10$ \\
\hline 3 & $6.707 \mathrm{e}-03$ & $3.706 \mathrm{e}-03$ & $2.269 \mathrm{e}-03$ & $1.515 \mathrm{e}-03$ \\
4 & $3.132 \mathrm{e}-04$ & $9.632 \mathrm{e}-05$ & $4.302 \mathrm{e}-05$ & $2.278 \mathrm{e}-05$ \\
4 & $4.164 \mathrm{e}-06$ & $8.987 \mathrm{e}-07$ & $2.980 \mathrm{e}-07$ & $1.266 \mathrm{e}-07$ \\
& $3.713 \mathrm{e}-07$ & $5.189 \mathrm{e}-08$ & $1.332 \mathrm{e}-08$ & $6.535 \mathrm{e}-09$ \\
\hline
\end{tabular}

Table 3 Approximation error for the discharge (top) and the pressure (bottom) varying the approximation order $r+1$ and the mesh size $h$. Values in boxes correspond to problems with a total number of degrees of freedom close to 1000. It is worth noting that for a fixed number of degrees of freedom, one gets a smaller error using high order methods.

choice of a direct solver is done to avoid the CPU uncertainty related to fixing precision thresholds or choosing preconditioning techniques for iterative algorithms.

In Table 3 we report the approximation error for both the discharge and the pressure in terms of the mesh size $h$ and of the polynomial degree $r+1$. It is the $L^{\infty}$-norm of the fields $\mathbf{q}_{e x}-\mathbf{q}_{h}$ and $p_{e x}-p_{h}$, evaluated at the barycentre of the small triangles. Let us recall that $\mathbf{q}_{h}=\sum_{j=1}^{\mathrm{N}_{0}} Q_{j} \mathbf{w}_{h, j} p_{h}=\sum_{j=1}^{\mathrm{M}} P_{j} \pi_{h, j}$. It is worth noting that for a fixed number of degrees of freedom, one gets smaller error using high order methods. Figure 5 shows graphically these data. It is easy to check that the convergence rate of the discharge in terms of $h$ is equal to the degree of the polynomials in the Raviart-Thomas space while the convergence rate of the pressure is even better than the expected one.

Using the tree-cotree approach, it is possible to decrease significantly the dimension of the system to be solved. In the case of a two dimensional domain with connected boundary and $\Gamma_{N}=\emptyset$ the dimension of the space of discontinuous piecewise polynomial functions of degree $r$ is $\mathrm{M}=(r+2)(r+1) n_{T} / 2$ 

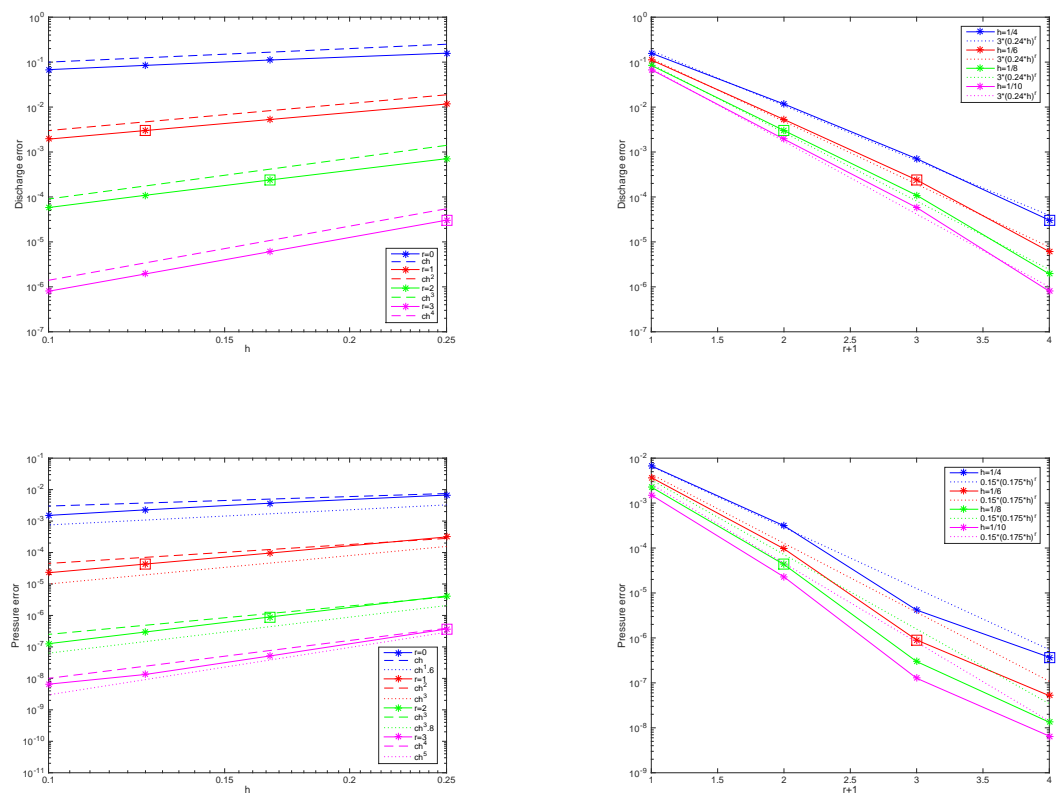

Fig. 5 Approximation error in terms of the mesh size $h$ for different values of $r$ (left) and in terms of the polynomial degree for differente values of $h$ (right). On the top the discharge, on the bottom the pressure. Marked values correspond to the values in boxes in Table 3

and the dimension of the space of Raviart-Thomas finite elements of degree $r+1$ is $N=N_{0}=(r+1) n_{F}+r(r+1) n_{T}$ being $n_{T}$ (resp., $\left.n_{F}\right)$ the total number of triangles (resp., of faces, that are edges in the two dimensional case) of the mesh. The indefinite linear system (10) has dimension $\mathrm{N}_{0}+\mathrm{M}$, whereas the matrix $\tilde{A}$ in system (20) has the dimension of the cotree, namely, $\mathrm{N}_{0}-\mathrm{M}$. However the computation of $\tilde{A}$ involves the inverse of the matrix $B_{s t}$ that has the dimension of the tree M.

The tree-cotree decomposition has been implemented in Maltab using the commands graph, dfsearch and bfsearch. The last two commands define a tree on a graph using a depth-first search (DFS) or a breadth-first search (BFS) respectively. For the study conducted in this work, the two searches are equivalent and have similar computational times.

Table 4 shows the computational time (in millisecond) for the two spanning trees when considering, in the top part, a fixed approximation order $r+1=3$ on different meshes, in the bottom part, a fixed mesh size $h=1 / 4$ with 


\begin{tabular}{|c|r|r|rr|rr|}
\hline & Number & Direct & \multicolumn{4}{|c|}{ Tree-Cotree decomp. } \\
& of dofs & solver & \multicolumn{2}{|c|}{ with DFS } & \multicolumn{2}{|c|}{ with BFS } \\
$(r+1, h)$ & & ms & $(\mathrm{R})$ & ms & $(\mathrm{R})$ & ms \\
\hline & & & & & & \\
$(3,1 / 2)$ & 144 & 0.9 & $(-13.55)$ & 13.1 & $(-13.2)$ & 12.8 \\
$(3,1 / 3)$ & 315 & 1.5 & $(-7.13)$ & 12.2 & $(-6.93)$ & 11.9 \\
$(3,1 / 4)$ & 552 & 7.0 & $(-1.41)$ & 16.9 & $(-1.30)$ & 16.1 \\
$(3,1 / 5)$ & 855 & 21.1 & $(0.16)$ & 17.6 & $(0.00)$ & 21.2 \\
$(3,1 / 6)$ & 1224 & 46.1 & $(0.39)$ & 28.1 & $(0.42)$ & 26.7 \\
$(3,1 / 7)$ & 1659 & 105.8 & $(0.67)$ & 34.4 & $(0.62)$ & 40.2 \\
$(3,1 / 8)$ & 2160 & 192.9 & $(0.68)$ & 60.9 & $(0.73)$ & 50.6 \\
\hline & & & & & & \\
$(1,1 / 4)$ & 88 & 0.6 & $(-23.83)$ & 14.9 & $(-22.83)$ & 14.3 \\
$(2,1 / 4)$ & 272 & 1.5 & $(-7.00)$ & 12.0 & $(-8.73)$ & 14.6 \\
$(3,1 / 4)$ & 552 & 7.0 & $(-1.41)$ & 16.9 & $(-1.30)$ & 16.1 \\
$(4,1 / 4)$ & 928 & 23.2 & $(0.21)$ & 18.2 & $(0.25)$ & 17.2 \\
\hline
\end{tabular}

Table 4 Computational time for the direct solver and tree-cotree method, when considering either the same approximation order $r+1=3$ on different meshes (top part) or the same mesh size $h=1 / 4$ and different approximation orders. In the second column it is reported the number of degrees of freedom of each test case and, in the third column the time (in millisecond) to solve the linear system with matrix $M$ using the Matlab backslash command. In the last two columns, we indicate the time (in millisecond) to solve the linear system relying on the tree-cotree decomposition with the depth-first and the breadth-first searches. In these two last columns, we also report the reduction index $(\mathrm{R})$.

\begin{tabular}{|c|c|c|c|c|c|c|c|c|c|c|}
\hline & \multirow{3}{*}{ dofs } & \multirow{3}{*}{$\begin{array}{l}\text { Direct } \\
\text { solver }\end{array}$} & \multicolumn{8}{|c|}{ Tree-Cotree decomposition } \\
\hline & & & \multicolumn{4}{|c|}{ with DFS } & \multicolumn{4}{|c|}{ with BFS } \\
\hline & & & $\mathrm{ST}$ & $\mathrm{IB}_{s t}$ & $\mathrm{IA}$ & Tot. & $\mathrm{ST}$ & $\mathrm{IB}_{s t}$ & $\mathrm{IA}$ & Tot. \\
\hline$(2,1 / 8)$ & 1056 & 26.0 & 15.1 & 6.4 & 3.5 & 25.0 & 15.0 & 7.4 & 2.2 & 24.7 \\
\hline$(3,1 / 6)$ & 1224 & 46.1 & 14.7 & 9.3 & 4.1 & 28.1 & 14.3 & 9.5 & 2.9 & 26.7 \\
\hline$(4,1 / 4)$ & 928 & 23.2 & 14.3 & 2.6 & 1.3 & 18.2 & 13.3 & 2.6 & 1.3 & 17.2 \\
\hline
\end{tabular}

Table 5 Computational time for the direct solver and tree-cotree method, when considering a given number of degrees of freedom (see the values in boxes of Table 3). The approximation order together with the mesh size and the number of degrees of freedom of each test case are reported in the first and second columns, respectively. In the third column the time (in millisecond) to solve using the Matlab backslash command the linear system with matrix $M$. The last eight columns concern the tree-cotree decomposition with the depth-first search and the breadth-first search. ST indicates the time to read the mesh and compute the spanning tree, $\mathrm{IB}_{s t}$ indicates the time to invert the matrix $B_{s t}$ and IA indicates the time to invert $\tilde{A}$. Tot indicates the total time, namely, the sum of the values ST, IB st $_{\text {and }} \tilde{\mathrm{A}}$ contained in the three previous columns.

different approximation orders. It also includes the computational time to solve the complete linear system with a direct method. The computational time is similar for the two choices of spanning tree. The BFS tree seems to be slightly more efficient. The solution of the discrete problem based on the presented tree-cotree decomposition is clearly faster than the direct solution of the linear system when the total number of degrees of freedom increases. One can indeed estimate the index of reduction, say $R$, on the cpu time necessary to 
solve the discrete problem when relying on the new tree-cotree decomposition. We have $R=\left(1-t_{r} / t_{c}\right)$, with $t_{r}$ (resp. $t_{c}$ ) the cpu time to solve the discrete problem with the new method (resp. with the classical method). By using the values presented in Table 4 for the case $r+1=3$ and $h \leq 1 / 5$ with DFS, we have $R$ increasing from 0.2 up to 0.7 (thus a cpu time reduction of $70 \%$ ).

In Table 5 we report the computational time (in millisecond) for the two spanning trees when considering a number of degrees of freedom similar to the ones associated with the values in boxes on Table 3. For the tree-cotree decomposition the computational time is divided into the time to read the mesh and compute the spanning-tree (ST), the time to compute $B_{s t}^{-1}\left(\mathrm{IB}_{s t}\right)$ and the time to solve the linear system with matrix $\tilde{A}$ (I $\tilde{A})$.

\section{Conclusions}

The term finite element exterior calculus refers to the finite element approximation of differential $k$-forms. In this framework the elements of the finite element spaces are studied as polynomial differential $k$-forms. It is then natural to use, as degrees of freedom, integrals on $k$-chains because, for differential $k$-forms, they have a clear physical meaning. The set of degrees of freedom considered in this work, the weights, are based on this idea. Using the weights many procedures and techniques that are well understood in the case of finite elements of minimal degree can be extended in a natural way to finite elements of higher degree. This advantage of the weights has been exploited in electromagnetic problems but, from our knowledge, not yet in fluid dynamics. In this work, taking advantage of the use of the weights, we implement a treecotree decomposition for the solution of the linear system resulting from the high-order Whitney approximation of Darcys flows.

We have presented the Darcy's problem in terms of differential forms in the FEEC framework, in order to show how naturally the continuous and discrete functional spaces can be selected when going back to vector and scalar fields. The two physical unknown quantities, the discharge and the pressure, are approximated using high order Whitney differential $(n-1)$ - and $n$-forms, that correspond to Raviart-Thomas finite elements of degree $r+1$ and discontinuous piecewise polynomial finite elements of degree $r$, respectively. Then the new degrees of freedom, the weights on the small simplices, have been considered. We have numerically estimated the inf-sup constant and shown that it is independent from $h$ and $r$, thus confirming the stability of the proposed method. Successively, to solve the discrete problem taking into account the divergence free constraint on the discharge, we have relied on a tree-cotree block-wise decomposition of the unknowns based on graph theory that is very natural when considering the weights on the small simplices as degrees of freedom. This because, being the weights of an $(n-1)$-form integrals on $(n-1)$-faces of $n$-simplices, the divergence matrix is the transpose of a boundary matrix also when $r>0$. This property is well-known and exploited in the case $r=0$ 
The proposed approach was meant for three-dimensional applications [3] (see for example [6], Fig. 1.4, for the construction of the dual graph in three dimensions). However for simplicity we have implemented it in Matlab in the two-dimensional case. Some numerical tests validate the interest in the proposed approach. When the computational mesh becomes fine, the use of the tree-cotree decomposition reduces significantly the computational time to solve the linear system. This method could be adopted in any FEM package that implements high order mixed finite elements.

Acknowledgements This research was supported by the program MathIT financed by the ANR-15-IDEX-01 of the Universite Côte Azur (UCA) in Nice, France. E. Zappon warmly thanks the Università degli Studi di Trento (Italy) for the possibility of studying as ERASMUS fellow at the Université Côte Azur, where this work began. The first author was partially supported by the project PRIN 201752HKH8.

The authors wish to dedicate this work to the memory of Christine Bernardi, research director at the CNRS. Numerical methods applied to Darcy's model was one of her favorite subjects.

\section{References}

1. Ainsworth, M., Andriamaro, G., Davydov, O.: A Bernstein-Bézier basis for arbitrary order Raviart-Thomas finite elements. Constr. Approx., 41, 1-22 (2015).

2. Alonso Rodríguez, A., Bruni Bruno, L., Rapetti, F.: Minimal sets of unisolvent weights for high order Whitney forms on simplices. Enumath 2019 procs., Lecture Notes in Comput. Sci. and Engng., Springer-Verlag (2020).

3. Alonso Rodríguez, A., Camaño, J., De Los Santos, E., Rapetti, F.: A graph approach for the construction of high order divergence-free Raviart-Thomas finite elements. Calcolo 55, 42 (2018)

4. Alonso Rodríguez, A., Camaño, J., De Los Santos, E., Rapetti, F.: A tree-cotree splitting for the construction of divergence-free finite elements: the high-order case. Submitted (2019), hal-02429500.

5. Alonso Rodríguez, A., Camaño, J., Ghiloni, R., Valli, A. : Graphs, spanning trees and divergence-free finite elements in domains of general topology. IMA J. Numer. Anal. 37 1986-2003 (2017).

6. Alonso Rodríguez, A., Rapetti, F.: Small trees for high order Whitney elements. Lecture Notes in Comput. Sci. and Engng., 134, Springer-Verlag, 587-597 (2020).

7. Alotto, P., Perugia, I.: Mixed finite element methods and tree-cotree implicit condensation. Calcolo, 36, 233-248 (1999).

8. Arnold, D.: Finite Element Exterior Calculus. SIAM (2018) isbn: 978-1-611975-53-6.

9. Arnold, D., Falk, R., Winther, R.: Finite element exterior calculus, homological techniques, and applications. Acta Numer., 15, 1-155 (2006).

10. Arnold, D., Falk, R., Winther, R.: Finite element exterior calculus: from Hodge theory to numerical stability. Bull. (New Series) of the American Math. Soc., 47, 281-354 (2010).

11. Bernardi, C., Maday, Y., Rapetti, F.: Discrétisations variationnelles de problèmes aux limites elliptiques. 310 pages. Mathématiques \& Applications, 45, Springer-Verlag (2004).

12. Boffi, D., Brezzi, F., Fortin, M.: Mixed finite element methods and applications. 685 pages. Springer Series in Comput. Math., 44 (2013).

13. Bonazzoli, M., Rapetti, F.: High order finite elements in numerical electromagnetism: degrees of freedom and generators in duality. Numer. Algorithms, 74, 111-136 (2017).

14. Bossavit, A.: Computational electromagnetism: variational formulations, complementarity, edge elements. 375 pages. Academic Press, New York (1998).

15. Chapelle, D., Bathe, K.J.: The inf-sup test. Comput. \& Structures, 47, 537-545 (1993).

16. Christiansen, S.H., Rapetti, F.: On high order finite element spaces of differential forms. Math. Comput., 85, 517-548 (2016). 
17. Cliffe, K.A., Graham, I.G., Scheichl, R., Stals, L.: Parallel Computation of Flow in Heterogeneous Media Modelled by Mixed Finite Elements, J. Comput. Phys., 164, 258 $282(2000)$.

18. Darcy, H.: Les fontaines publiques de la ville de Dijon. 647 pages. Victor Dalmont ed., Paris (1956)

19. Dubois, F.: Discrete vector potential representation of a divergence-free vector field in three-dimensional domains: numerical analysis of a model problem, SIAM J. Numer. Anal. 27, 1103-1141 (1990).

20. Ern, A., Guermond, J.-L.: Finite elements II: Galerkin Approximation, Elliptic and Mixed PDEs. Vol. 73, Texts in Applied Mathematics, Springer (2020).

21. Fortin, M., Pierre, R.: Stability Analysis of Discrete Generalized Stokes Problem. Numer. Meth. Partial Differ. Eq., 3, 303-323 (1992).

22. Freeze, R.A., Cherry, J.A.: Groundwater. 604 pages. Prentice Hall (1979).

23. Hecht, F.: Construction d'une base de fonctions P1 non conforme à divergence nulle dans $\mathbb{R}^{3}$. RAIRO Anal. Numer. 15, 119-150 (1981).

24. Hirani, A.N., Nakshatrala, K.B., Chaudhry, J.H.: Numerical method for Darcy flow derived using discrete exterior calculus. Int. J. Comp. Meth. Engng. Sci. Mech. 16, 151169 (2015).

25. Kuznetsov, N., Nazarov, A.: Sharp constants in the Poincaré, Steklov and related inequalities (A survey). Matematika, 1-17 (2014).

26. Lions, J.L., Magenes, E.: Non-homogeneous Boundary value problems and applications I. Springer-Verlag, New York (1972).

27. Nédélec, J.-C.: Mixed finite elements in $\mathbb{R}^{3}$. Numer. Math., 35, 315-341 (1980).

28. Quarteroni, A., Valli, A.: Numerical approximation of partial differential equations. 551 pages. Springer Series in Comput. Math., 13 (1994).

29. Rapetti, F., Bossavit, A.: Whitney forms of higher degree. SIAM Numer. Anal., 47, 2369-2386 (2009).

30. Rapetti, F., Dubois, F., Bossavit, A.: Discrete vector potentials for non-simply connected three-dimensional domains. SIAM Numer. Anal. 41/4, 1505-1527 (2003).

31. Raviart, P.-A., Thomas, J.-M. A mixed finite element method for 2nd order elliptic problems. Mathematical aspects of finite element methods. (Proc. Conf. Consiglio Naz. delle Ricerche, Rome, 1975), 292-315. Lecture Notes in Math., Vol. 606, Springer, Berlin (1977)

32. Scheichl, R.: Decoupling Three-Dimensional Mixed Problems Using Divergence-Free Finite Elements. SIAM J. Sci. Comput., 23, 1752-1776 (2002).

33. Thulasiraman, K., Swamy, M. : Graphs. Theory and algorithms. A Wiley-Interscience Publication, John Wiley \& Sons, Inc., New York (1992).

34. Tonti, E.: On the mathematical structure of a large class of physical theories, Rend. Acc. Lincei, 52, 48-56 (1972).

35. Whitney, H.: Geometric integration theory. 400 pages. Princeton University Press (1957) 